\title{
Doping dependence of $d$-wave bond-charge excitations in electron-doped cuprates
}

\author{
Hiroyuki Yamase ${ }^{\ddagger}$, Matías Bejas ${ }^{\dagger}$, and Andrés Greco $^{\dagger}$ \\ ${ }^{\ddagger}$ National Institute for Materials Science, \\ Tsukuba 305-0047, Japan \\ ${ }^{\dagger}$ Facultad de Ciencias Exactas, \\ Ingeniería y Agrimensura and Instituto \\ de Física Rosario (UNR-CONICET), \\ Av. Pellegrini 250, \\ 2000 Rosario, Argentina
}

(Dated: January 31, 2019)

\begin{abstract}
Motivated by the recent experiments reporting the doping dependence of the short-range charge order $(\mathrm{CO})$ in electron-doped cuprates, we study the resonant x-ray scattering spectrum from $d$ wave bond-charge fluctuations obtained in the two-dimensional $t$ - $J$ model. We find that (i) the $\mathrm{CO}$ is short-range, (ii) the CO peak is pronounced at low temperature, (iii) the peak intensity increases with decreasing carrier doping $\delta$ down to $\delta \approx 0.10$ and is substantially suppressed below $\delta \approx 0.10$ due to strong damping, and (iv) the momentum of the CO decreases monotonically down to $\delta \approx 0.10$ and goes up below $\delta \approx 0.10$. These results reasonably capture the major features of the experimental data, and the observed short-range $\mathrm{CO}$ can be consistently explained in terms of bond-charge fluctuations with an internal $d$-wave symmetry.
\end{abstract}

PACS numbers: 


\section{INTRODUCTION}

Recently resonant x-ray scattering (RXS), resonant inelastic x-ray scattering (RIXS), and high-energy x-ray scattering revealed the presence of short-range charge order (CO) with modulation vector along the axial direction $(0,0)-(\pi, 0)$ in various hole-doped cuprates (h-cuprates) such as $\mathrm{Y}^{-1-5}, \mathrm{Bi}^{-}{ }^{6-9}$, and Hg-based ${ }^{10,11}$ compounds, implying that the CO can be a universal phenomenon in h-cuprates. The understanding of the origin of those charge correlations, therefore, will likely yield an important clue to the origin of the pseudogap as well as high- $T_{c}$ superconductivity ${ }^{12}$. In fact, a large number of theoretical studies were performed ${ }^{13-22}$, although a consensus has not been obtained.

On the other hand, a short-range CO was also observed in electron-doped cuprates ${ }^{23-25}$ (e-cuprates). Since the pseudogap features similar to those in h-cuprates are much weaker in e-cuprates, a theoretical study may be less complicated in e-cuprates. However, compared to theoretical studies of h-cuprates ${ }^{13-22}$, the CO in e-cuprates is much less studied ${ }^{26-29}$. Ref. 26 showed a comprehensive study of all possible COs in the two-dimensional (2D) $t$ - $J$ model and found a strong tendency to $d$-wave bond-charge order ${ }^{30}$. Ref. 27 then showed that $d$-wave bond-charge fluctuations can capture the charge excitation spectrum observed in experiments $^{23}$. Although the theoretical framework is different from Refs. 26 and 27, similar $d$-wave bond-charge fluctuations were also proposed in Ref. 28 to explain the experimental data.

The $d$-wave bond-charge order is different from a usual textbook-like charge-densitywave because the bond charge has an internal structure characterized by a $d$-wave symmetry. Therefore if the short-range CO observed in e-cuprates is indeed a $d$-wave bond-charge order, it can be interpreted as the first observation of unconventional CO in e-cuprates. Given that $d$-wave bond-charge order was discussed in h-cuprates ${ }^{31}$, it can be a universal phenomenon in the whole cuprate family. In addition, $d$-wave bond-charge order would be reduced to the electronic nematic order ${ }^{32}$, more precisely a $d$-wave Pomeranchuk instability ${ }^{33-35}$ when the momentum transfer approaches zero. In this sense, the nematic physics can also play a role in the charge dynamics in e-cuprates while the nematic physics is discussed only in h-cuprates so far ${ }^{36-39}$. Therefore it is very important to study whether recent experimental data in e-cuprates ${ }^{24}$, i.e., the doping dependence of the short-range CO, can be indeed captured in terms of $d$-wave bond charge, which will then provide a crucial step to establish 
the presence of $d$-wave bond-charge fluctuations in e-cuprates.

In this paper, we compute the static charge susceptibility associated with $d$-wave bondcharge order and then the equal-time correlation function $S(\mathbf{q})$, the quantity measured by RXS, in a large- $N$ scheme of the $t-J$ model. We show that our theoretical results capture the major features of the doping dependence of the recent RXS data ${ }^{24}$. Our calculations suggest the presence of unconventional charge fluctuations in e-cuprates, which are connected with the nematic order in the limit of momentum to zero.

\section{MODEL AND FORMALISM}

The $d$-wave bond charge is obtained in a non-bias study of the $2 \mathrm{D} t-J$ model by employing a large- $N$ scheme ${ }^{13}$. We follow such a theoretical scheme and focus on the excitation spectrum of the $d$-wave bond charge.

The $2 \mathrm{D} t-J$ model on a square lattice reads

$$
H=-\sum_{i, j, \sigma} t_{i j} \tilde{c}_{i \sigma}^{\dagger} \tilde{c}_{j \sigma}+J \sum_{\langle i, j\rangle}\left(\vec{S}_{i} \cdot \vec{S}_{j}-\frac{1}{4} n_{i} n_{j}\right)+V \sum_{\langle i, j\rangle} n_{i} n_{j}
$$

where $t_{i j}=t\left(t^{\prime}\right)$ is the hopping between the first (second) nearest-neighbors sites; $J$ and $V$ are the magnetic exchange and Coulomb interaction between the nearest-neighbors sites as denoted by $\langle i, j\rangle$, respectively. $\tilde{c}_{i \sigma}^{\dagger}\left(\tilde{c}_{i \sigma}\right)$ is the creation (annihilation) operators of electrons with spin $\sigma(=\uparrow, \downarrow)$ in the Fock space without any double occupancy. $n_{i}=\sum_{\sigma} \tilde{c}_{i \sigma}^{\dagger} \tilde{c}_{i \sigma}$ is the electron density operator and $\vec{S}_{i}$ is the spin operator.

Various approximations to the $t-J$ model $^{26,40,41}$ found a strong tendency toward phase separation especially for band parameters appropriate for e-cuprates. The phase separation, however, can be an artifact caused by discarding the Coulomb repulsion ${ }^{42}$. Therefore we included the nearest-neighbor Coulomb interaction in the Hamiltonian (1) to suppress the tendency of the phase separation. From a more realistic point of view, we would include the interlayer hopping as well as the long-range Coulomb interaction. This is actually important when studying the high-energy charge excitation spectrum, which is dominated by plasmon excitations ${ }^{43-45}$. However, three dimensionality and the precise form of the Coulomb interaction are not important to low-energy charge excitations ${ }^{44}$ addressed in the present work. 
It is not straightforward to analyze the Hamiltonian (1) because it is defined in the Fock space without double occupancy of electrons. Here we employ a large- $N$ technique in a path integral representation of the Hubbard $X$ operators ${ }^{13,46}$, where the leading order approximation becomes exact in the limit of large $N$. With this formalism, all possible charge instabilities included in the Hamiltonian (1) are treated on equal footing and were studied at leading order ${ }^{13,26}$. In particular, for band parameters appropriate for e-cuprates, $d$-wave bond-charge instability is leading around the doping rate $\delta=0.15$; see Figs. 4(b) and 6 in Ref. 26 for the phase diagram. As seen in the phase diagram, there are other $\mathrm{CO}$ tendencies close to $d$-wave bond-charge order and their low-energy charge excitations are actually present; see Fig. 7 in Ref. 44. However, other charge excitations do not show a possible softening along the $(0,0)-(\pi, 0)$ direction and cannot capture the experimental data $^{23}$. In fact, it is only $d$-wave bond charge which exhibits a peak structure along the $(0,0)-(\pi, 0)$ direction in $S(\mathbf{q})$ as shown in Fig. 6 in Ref.44 and captures ${ }^{27}$ the essential features of the experimental data in e-cuprates at $\delta=0.14$ and 0.15 (Ref. 23). Therefore to study the recent experimental data performed at different doping rates in e-cuprates ${ }^{24}$, we focus on the charge excitations coming from $d$-wave bond-charge fluctuations. Since we deal with the $t-J$ model, which is derived from the three-band Hubbard model ${ }^{47}$, bond-charge order can be interpreted as charge-density-wave at the oxygen sites because bond charge is defined on a bond between the nearest-neighbor $\mathrm{Cu}$ sites.

RXS measures the equal-time correlation function, which is defined by

$$
S(\mathbf{q})=\frac{1}{\pi} \int_{-\omega_{c}}^{\omega_{c}} \mathrm{~d} \omega \operatorname{Im} \chi_{d}(\mathbf{q}, \omega)\left[n_{B}(\omega)+1\right],
$$

where $n_{B}(\omega)=1 /\left(\mathrm{e}^{\omega / T}-1\right)$ is the Bose factor and $T$ is temperature. We introduced the cutoff energy $\omega_{c}$ for a later convenience (see Fig. 5) and $\omega_{c}=\infty$ for the equal-time correlation function. The $d$-wave bond-charge susceptibility $\chi_{d}(\mathbf{q}, \omega)$ is obtained in the large- $N$ expansion at leading order as ${ }^{13}$

$$
\chi_{d}(\mathbf{q}, \omega)=\frac{\left(8 J \Delta^{2}\right)^{-1}}{1-2 J \Pi_{d}(\mathbf{q}, \omega)},
$$

which becomes exact in the limit of large $N$. Here $\Delta$ is the mean-field value of a bond field and is given by $\Delta=\frac{1}{4 N_{s}} \sum_{\mathbf{k}}\left(\cos k_{x}+\cos k_{y}\right) f\left(\epsilon_{\mathbf{k}}\right)$; the value of $\Delta$ is determined selfconsistently. This bond field $\Delta$ naturally appears in our path integral formalism ${ }^{13}$ as a Hubbard-Stratonovich field. $N_{s}$ is the total number of lattice sites and $f(x)=1 /\left(\mathrm{e}^{x / T}+1\right)$ 
is the Fermi-Dirac distribution function. The electron dispersion $\epsilon_{\mathbf{k}}$ is renormalized already at leading order in the large- $N$ expansion

$$
\epsilon_{\mathbf{k}}=-2\left(t \frac{\delta}{2}+J \Delta\right)\left(\cos k_{x}+\cos k_{y}\right)-4 t^{\prime} \frac{\delta}{2} \cos k_{x} \cos k_{y}-\mu,
$$

where $t$ and $t^{\prime}$ are reduced by a factor of $\delta / 2$ and $\mu$ is the chemical potential. The $d$-wave polarization $\Pi_{d}(\mathbf{q}, \omega)$ in Eq. (3) reads

$$
\Pi_{d}(\mathbf{q}, \omega)=-\frac{1}{N_{s}} \sum_{\mathbf{k}} \gamma^{2}(\mathbf{k}) \frac{f\left(\epsilon_{\mathbf{k}+\mathbf{q} / 2}\right)-f\left(\epsilon_{\mathbf{k}-\mathbf{q} / 2}\right)}{\epsilon_{\mathbf{k}+\mathbf{q} / 2}-\epsilon_{\mathbf{k}-\mathbf{q} / 2}-\omega-\mathrm{i} \Gamma},
$$

where the $d$-wave form factor $\gamma(\mathbf{k})=\left(\cos k_{x}-\cos k_{y}\right) / 2$ describes a $d$-wave symmetry of the bond-charge order and $\Gamma$ is infinitesimally small. In the limit of $\mathbf{q}=\mathbf{0}, \chi_{d}(\mathbf{q}, \omega)$ would be reduced to the electronic nematic susceptibility ${ }^{48}$ associated with a $d$-wave Pomeranchuk instability $^{33-35}$. In the following, we measure all quantities with the dimension of energy in units of $t$. A realistic value of $t / 2$ (Ref. 49) in cuprates is around $500 \mathrm{meV}$ (Ref. 50),

\section{RESULTS}

We choose $J=0.3$ and $t^{\prime}=0.3$ in our Hamiltonian (1) as typical parameters for ecuprates $^{26}$; the precise value of $V$ is not important as long as it suppresses phase separation. As a value of $\Gamma$ in Eq. (5) we take $\Gamma=10^{-4}$, which is reasonably small. In Fig. 1(a), we present the static part of the $d$-wave bond-charge susceptibility $\chi_{d}(\mathbf{q})=\chi_{d}(\mathbf{q}, \omega=0)$ as a function of $\mathbf{q}$ for several choices of temperatures at $\delta=0.13$. Note that $\chi_{d}(\mathbf{q})$ has $4 \pi$ periodicity because of the presence of the $d$-wave form factor [see Eq. (5)] and thus the $\mathbf{q}$ region is in $0 \leq q_{x} \leq 2 \pi$ in Fig. 1(a). With decreasing $T$, a peak is pronounced at $\mathbf{q}=\left( \pm 2 \pi Q_{\mathrm{co}}, 0\right)$ and $\left(0, \pm 2 \pi Q_{\mathrm{co}}\right)$ with $Q_{\mathrm{co}} \approx 0.25$, indicating a tendency toward a charge ordered phase. However, the static susceptibility does not diverge and the CO remains a short range.

We show in Fig. 1(b) the equal-time correlation function $S(\mathbf{q})$ for the same parameters as in Fig. 1(a). While $S(\mathbf{q})$ has a peak at almost the same position of $\chi_{d}(\mathbf{q})$, the peak intensity is slightly suppressed with decreasing $T$ (Ref. 51) even though $\chi_{d}(\mathbf{q})$ shows a pronounced peak at low $T$ [Fig. 1(a)]. This counter-intuitive feature comes from the presence of the Bose factor $n_{B}(\omega)$ in Eq. (2). In fact, if $n_{B}(\omega)$ were omitted in Eq. (2), $S(\mathbf{q})$ would show a peak, which is enhanced with decreasing $T$. 


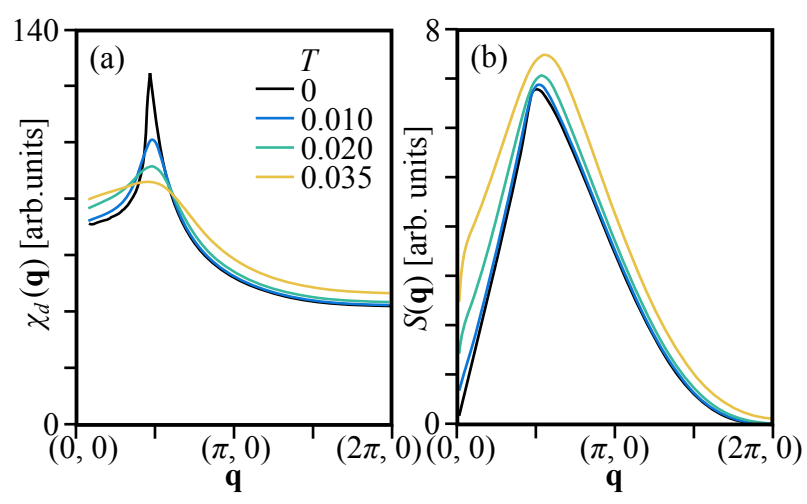

FIG. 1: (Color online) (a) q dependence of $\chi_{d}(\mathbf{q})$ for various choices of temperatures at $\delta=0.13$. (b) $S(\mathbf{q})$ for the same parameters as in (a) and the cutoff energy is $\omega_{c}=\infty$.

The suppression of $S(\mathbf{q})$ with decreasing $T$ in Fig. 1(b) can be an artifact because we completely neglect the damping of quasiparticles, that is, we assume $\Gamma=10^{-4}$ at any temperature. Apparently this is unphysical. In fact, a relatively large $\Gamma$ is frequently assumed when a spectral line shape obtained theoretically is compared with experimental data ${ }^{43,45,52}$. For example, in Ref. 43 a comparison to the experimental data was made successfully by assuming that $\Gamma$ increases with increasing temperature to understand the temperature dependence of the high-energy charge-excitation spectrum. Similarly, the damping effect should be pronounced also in a low doping region because of strong antiferromagnetic fluctuations there in cuprates. Therefore, we invoke a finite value of $\Gamma$ in Eq. (5) to simulate phenomenologically the damping of quasiparticles as a broadening of the spectrum.

A value of $\Gamma$ may in principle depend on $T, \delta, \mathbf{q}, \omega$, and others. Since our major interest is a study of temperature and doping dependences of $S(\mathbf{q})$, we consider only possible $T$ and $\delta$ dependences of $\Gamma$. As a function of $T$, a leading correction may be given by a linear term in $T$ (Ref. 53), i.e.,

$$
\Gamma(\delta, T)=\Gamma(\delta)+\alpha T
$$

Concerning the doping dependence, we recall that neutron scattering experiments ${ }^{54}$ revealed that the antiferromagnetic correlation length starts to increase substantially below $\delta \approx 0.10$ in the normal metallic phase around $T \sim 300 \mathrm{~K}$. Concomitantly, quasiparticles may be damped heavily below $\delta \approx 0.10$. To mimic this phenomenology in a simple way, we assume a $\delta$ dependence of $\Gamma(\delta)$ as shown in Fig. 2, where $\Gamma$ increases rapidly below $\delta \approx 0.10$. An 
explicit expression is given by

$$
\Gamma(\delta)=0.001+0.05\left[1-\tanh \left(\frac{\delta-0.09}{0.02}\right)\right] .
$$

In Fig. 2 we also plot the boundary of the $d$-wave bond-charge phase at $T=0$. When $\Gamma$ is infinitesimally small and independent of doping, the model would exhibit the $d$-wave bond-charge instability at $\delta_{c} \approx 0.125$ (Ref. 26 and also see Appendix). With increasing $\Gamma$, the $d$-wave bond-charge phase shrinks. As a result, we have only charge fluctuations associated with the $d$-wave bond-charge order for doping above the dashed line in Fig. 2.

While the choice of the absolute value of $\Gamma$ is rather arbitrary in Eq. (7), we choose it to have no charge instabilities even at low doping rate at $T=0$ (solid line in Fig. 2), so that our calculations are performed in the paramagnetic phase in the entire doping region. For a finite $T$, we choose $\alpha=9$ in Eq. (6) after checking that our conclusions are not modified for other choices of $\alpha=3$ and 6 . While we specified the functional form of $\Gamma$ [Eqs. (6) and (7)] to perform systematic calculations, the precise functional form itself is not important as long as $\Gamma$ increases with increasing temperature and decreasing doping, so that charge instabilities (see Appendix for details) are suppressed in a low doping region.

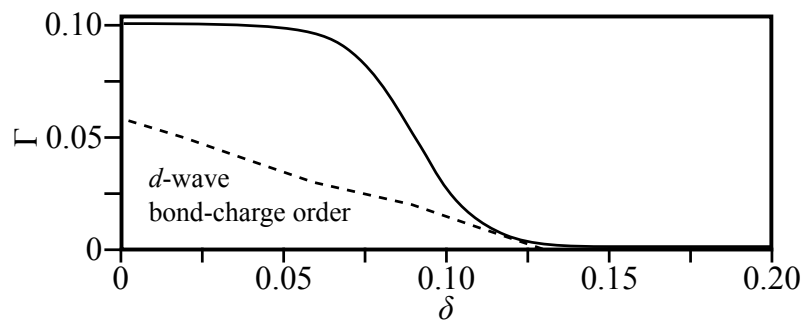

FIG. 2: Doping dependence of the damping $\Gamma$ (solid line) and the phase boundary of the $d$-wave bond-charge order at $T=0$ (dashed line).

Figure 3(a) shows $S(\mathbf{q})$ along the direction $(0,0)-(2 \pi, 0)$ in a temperature range $0<$ $T<0.035$ for $\delta=0.13$. $S(\mathbf{q})$ forms a peak structure at $\mathbf{q} \sim(0.5 \pi, 0)$ even at high $T$. To clarify how the peak of $S(\mathbf{q})$ develops, we define $\Delta S(\mathbf{q})=S(\mathbf{q} ; T)-S(\mathbf{q} ; T=0.035)$. Since $T=0.035$ corresponds to $T \approx 400 \mathrm{~K}$, the intensity at $T=0.035$ may be regarded as a background. Hence $\Delta S(\mathbf{q})$ is regarded as $S(\mathbf{q})$ after background subtraction, as often seen in an experimental data analysis ${ }^{24}$. We plot in Fig. 3(b) the temperature dependence of the peak intensity of $\Delta S(\mathbf{q})$ for several choices of doping rates $(>0.10)$. The peak intensity 

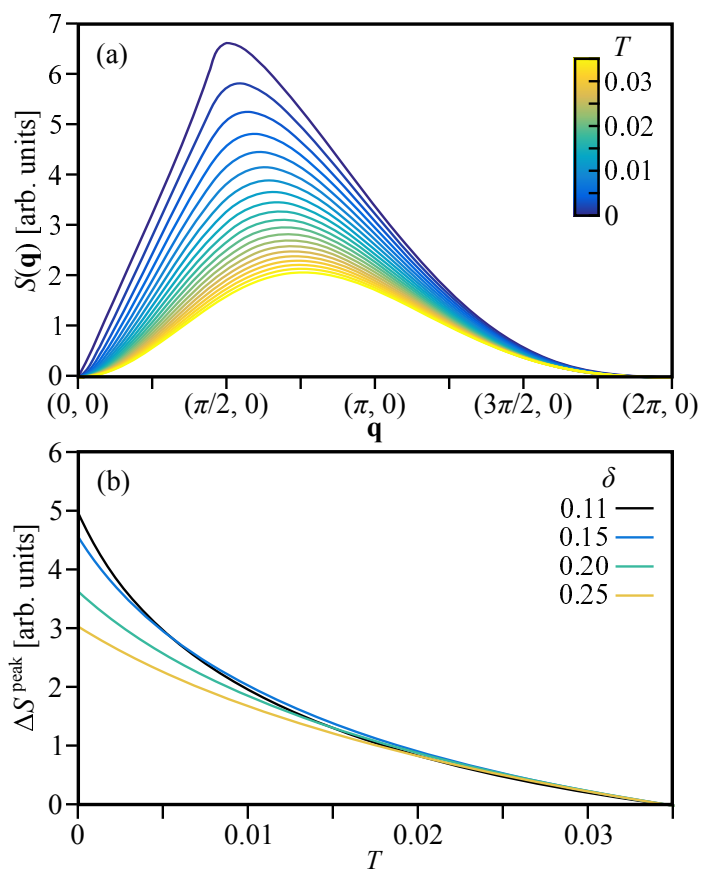

FIG. 3: (Color online) (a) q dependence of $S(\mathbf{q})$ for various choices of temperatures at doping $\delta=0.13$. (b) $\Delta S^{\text {peak }}$ as a function of temperature for several choices of doping rates. The cutoff energy is $\omega_{c}=\infty$.

$\Delta S^{\text {peak }}$ is pronounced upon decreasing temperature and doping rate, which is qualitatively the same as the experimental observation (see Fig. 2 G in Ref. 24).

With decreasing $\delta$ beyond $\delta \approx 0.10, S(\mathbf{q})$ is suppressed substantially as shown in Fig. 4, which is actually observed in experiments ${ }^{24}$. This rapid suppression comes from the pronounced increase of the damping $\Gamma$ below $\delta \approx 0.10$ as shown in Fig. 2. If $\Gamma$ is assumed to be constant, the peak intensity of $S(\mathbf{q})$ would continue to increase with decreasing $\delta$.

As shown in Fig. $3(\mathrm{a}), S(\mathbf{q})$ exhibits a peak at $\mathbf{q}=\left( \pm 2 \pi Q_{\mathrm{co}}, 0\right)$ and $\left(0, \pm 2 \pi Q_{\mathrm{co}}\right)$. This peak position is plotted in Fig. 4 as a function of doping rate at $T=0$ together with $Q_{\text {edge }}$, the distance between the Fermi surface edges across $\mathbf{k}=(\pi, 0)$ and its equivalent wavevectors (see the inset in Fig. 4). As pointed out in Ref. 27, the peak structure is formed by particle-hole scattering processes characterized by $Q_{\text {edge }}$. Hence $Q_{\text {co }}$ corresponds to such scattering wavevector $Q_{\text {edge }}$ at least down to $\delta \approx 0.10$, although it becomes slightly larger than $Q_{\text {edge }}$, since $S(\mathbf{q})$ is an energy-integrated quantity [see Eq. (2)]. Below $\delta \approx 0.10, Q_{\text {co }}$ goes up and deviates substantially from $Q_{\text {edge }}$. This is because the damping $\Gamma$ increases rapidly and the structure coming from the underlying Fermi surface is blurred. 


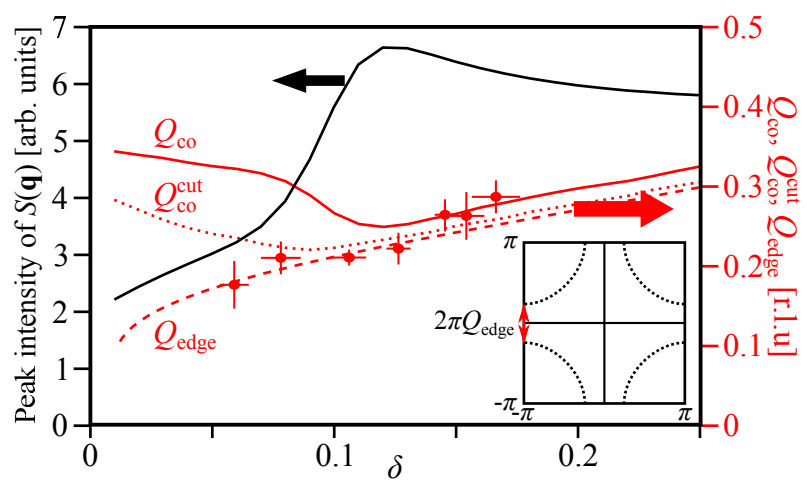

FIG. 4: (Color online) Peak intensity of $S(\mathbf{q})$ with $\omega_{c}=\infty$ and the momenta $Q_{\mathrm{co}}, Q_{\mathrm{co}}^{\text {cut }}$, and $Q_{\text {edge }}$ as a function of $\delta$ at $T=0 . Q_{\text {co }}$ is the peak position of $S(\mathbf{q})$ with $\omega_{c}=\infty$ whereas $Q_{\mathrm{co}}^{\text {cut }}$ is that of $S(\mathbf{q})$ with $\omega_{c}=0.05 ; Q_{\text {edge }}$ is defined in the inset. Solid circles are the experimental data from Ref. 24.

The peak width of $S(\mathbf{q})$ in Fig. 3(a) is very large even at $T=0$. This broadness is not due to our introduction of a finite $\Gamma$ [Eqs. (6) and (7)]. In fact, the peak of $S(\mathbf{q})$ is very broad even for $\Gamma=10^{-4}$ as shown in Fig. 1(b), although the static susceptibility exhibits a sharp peak at $\mathbf{q}=(0.5 \pi, 0)$ at low $T$. This counter-intuitive feature originates from the $\omega$ integration in Eq. (2). To demonstrate this, we decrease the cutoff energy $\omega_{c}$ in Eq. (2). As shown in Fig. 5(a), the resulting spectrum exhibits a shaper peak around $\mathbf{q}=(0.5 \pi, 0)$ for lower $\omega_{c}$. That is, the sharp peak of $S(\mathbf{q})$ for low $\omega_{c}$ originates from the short-range $d$-wave bond-charge order and this sharp peak is simply smeared out by spectral weight coming from the high-energy region. To extract direct contributions from the short-range $d$-wave bond-charge order, therefore, it may make sense to consider $S(\mathbf{q})$ with a low cutoff energy $\omega_{c}$, as actually done in a recent experimental analysis ${ }^{25}$. In Fig. 5(b), we plot $S(\mathbf{q})$ for $\omega_{c}=0.05$ for various choices of temperatures. $S(\mathbf{q})$ exhibits a broad spectrum at high $T$, but a sharp peak gradually grows below $T \sim 0.02$ as a consequence of development of a short-range bond-charge order. Similar to Fig. 3(b), we plot $\Delta S^{\text {peak }}$ as a function of $T$ for several choices of doping in the inset of Fig. 5(b). The overall feature is the same as Fig. 3(b), that is, the peak intensity increases at lower $T$ and for lower doping, consistent with the experiments ${ }^{24}$. Denoting the peak position of $S(\mathbf{q})$ in Fig. $5\left(\right.$ b) by $Q_{\text {co }}^{\text {cut }}$, we plot its doping dependence in Fig. 4. $Q_{\text {co }}^{\text {cut }}$ tends to follow $Q_{\text {edge }}$ down to rather low doping and starts to go upward below $\delta \approx 0.1$. 

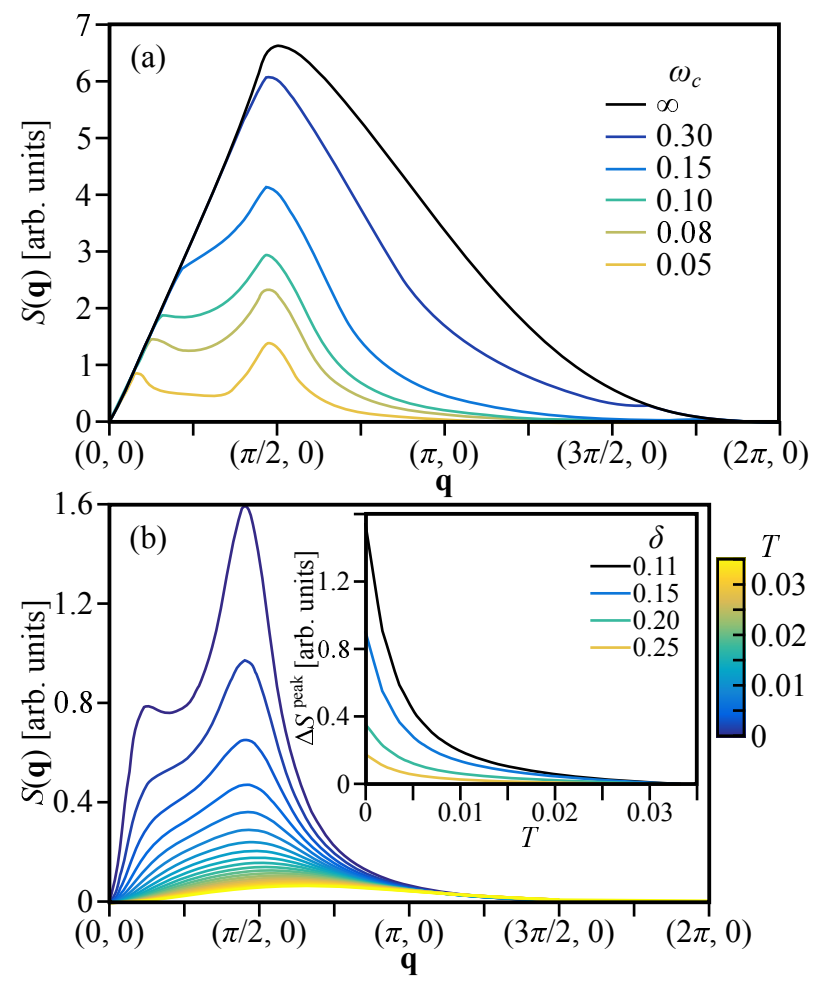

FIG. 5: (Color online) (a) q dependence of $S(\mathbf{q})$ for several choices of the cutoff energy at $\delta=0.13$ and $T=0$. (b) $\mathbf{q}$ dependence of $S(\mathbf{q})$ with a cutoff energy $\omega_{c}=0.05$ for various choices of temperatures at $\delta=0.13$. The inset shows the temperature dependence of the peak intensity of $\Delta S$ for several choices of doping rate after subtraction of intensity at $T=0.035$.

\section{DISCUSSIONS}

The equal-time correlation function $S(\mathbf{q})$ can be measured directly by RXS. In particular, we computed $S(\mathbf{q})$ associated with the $d$-wave bond-charge order, which is reduced to the nematic order in the limit of $\mathbf{q}=\mathbf{0}$ (Refs. 33-35). A possibility of $d$-wave bond-charge order $^{31}$ as well as the nematic order ${ }^{36-39}$ is already discussed in h-cuprates. Therefore it is a crucial step toward the understanding of the charge dynamics in cuprates to clarify to what extent our results capture recent RXS data in e-cuprates ${ }^{23-25}$. Since the previous data of Ref. 23 were already discussed in Ref. 27, we focus on the recent experimental data reported in Refs. 24 and 25.

The peak width of $S(\mathbf{q})$ shown in Fig. 3(a) is much larger than the experimental one ${ }^{24}$. However, when we focus on a low-energy region as shown in Fig. 5(b), the peak width becomes substantially small especially below $T \sim 0.01(\approx 100 \mathrm{~K})$ and comparable to the 
experimental data ${ }^{24}$. This suggests that compared with experiments, our results in Fig. 3(a) emphasize too much the contributions from high-energy spectral weight in $S(\mathbf{q})$ [Eq. (2)]. We consider two reasons for that. First, the spectral weight in a high-energy region in experiments can be much weaker than the present results and thus our results overestimates such a contribution, leading to a much broader peak. Second, as found in Ref. 25, the spectral weight reported in Refs. 23 and 24 comes not only from charge excitations, but also from magnetic excitations. In particular, the magnetic contribution extends from 200 to 700 meV at $Q_{\text {co }}$ and is pronounced below $T \sim 300 \mathrm{~K}$. These contributions are surely important to the resulting line shape of the spectrum, but are fully neglected in the present theory.

Nevertheless, our results are in line with the recent RIXS measurements. In Ref. 25, the spectral weight related to the CO signal is discussed to come from energy below $60 \mathrm{meV}$, whereas our sharp peak is realized in Fig. $5(\mathrm{a})$ for $\omega_{c} \approx 0.05-0.1$, which corresponds to 50 $100 \mathrm{meV}$. A possible reason why the spectral weight of charge excitations concentrates only in the low-energy region in experiments is that the actual system is located much closer to the vicinity of $\mathrm{CO}$ phase than the present theoretical situation (see Fig. 2).

The experimental observation that the energy range of charge dynamics coincides with that of magnetic excitations ${ }^{25}$ is also in line with our theory based on $d$-wave bond-charge order. As shown in Refs. 13 and 44, $d$-wave bond-charge order comes from the exchange interaction, i.e., the $J$-term in the $t$ - $J$ model. This is also clear from Eq. (3) where the interaction strength $J$ enters. If the charge dynamics originates from usual on-site charge excitations, its major contribution would appear in a higher energy region as plasmons ${ }^{43-45}$. On the other hand, needless to say, magnetic excitations are controlled by the $J$-term. Therefore both bond-charge and magnetic excitations naturally appear in the same energy scale of $J$. Obviously more theoretical studies are necessary when one wishes to address more details: for example, the reason why the magnetic excitations are strongest around $\omega=0.2$ $\mathrm{eV}$ whereas the typical charge excitation energy is lower than that, and why the magnetic excitations are enhanced at the same wavevector as that of the bond-charge excitations ${ }^{25}$. These issues are beyond the scope of the present work.

The peak position of $Q_{\text {co }}$ tends to be larger with increasing $T$ as shown in Fig. 3(a) whereas the peak position is almost independent of $T$ in the experiments ${ }^{24}$. However, given that the magnetic excitations are included in $S(\mathbf{q})$ mainly in a high-energy part in the experiments ${ }^{24}$, a comparison to the experiments may be made better by focusing on a low-energy region as 
shown in Fig. 5(b). In fact, in this case, our peak position is almost independent of $T$ below $T \sim 0.01$, which corresponds to about $100 \mathrm{~K}$.

$Q_{\text {co }}$ decreases with decreasing doping in the experiments ${ }^{24}$ (the experimental data are plotted in Fig. 4). In particular, it tends to be constant in $\delta \lesssim 0.10$ if we do not consider seriously the data at $\delta=0.059$, where the existence of a peak at $Q_{\text {co }}$ in Fig. 2(B) of Ref. 24 is unclear. We think that our theoretical results shown in Fig. 4 reasonably capture the major feature of $Q_{\mathrm{co}}$ observed in the experiments. In particular, if we consider $Q_{\mathrm{co}}^{\text {cut }}$, instead of $Q_{\mathrm{co}}$, the agreement with the experimental data is more satisfactory in a wide doping region.

As shown in Fig. 4, the peak intensity of $S(\mathbf{q})$ gradually increases and sharply drops in $\delta \lesssim 0.10$. Therefore the CO signal is expected to become difficult to be detected in $\delta \lesssim 0.10$. Conversely, we expect that the $\mathrm{CO}$ signal is observed more clearly above $\delta \approx 0.10$, as is indeed the case in the experiments ${ }^{24}$.

In the region above $\delta \approx 0.10$, the peak intensity of $S(\mathbf{q})$ increases at lower temperature and decreases for higher doping as shown in the inset of Fig. 5(b). This tendency is the same even if we take $\omega_{c}=\infty$ and include high-energy contribution to $S(\mathbf{q})$ [Fig. 3(b)]. Those temperature and doping dependence are consistent with the experimental observation ${ }^{24}$. Although the peak intensity in Fig. 5(b) seems to develop below a certain temperature, it should not be interpreted as the onset of the $d$-wave bond-charge order because the static susceptibility does not diverge down to zero temperature [see Fig. 1(a)].

We have shown that $d$-wave bond-charge order can reasonably capture the major features of low-energy charge excitations in e-cuprates ${ }^{23-25}$. What about other types of CO? The crucial points in the experimental data are twofold: (a) the CO tendency is detected along the $(0,0)-(\pi, 0)$ direction and (b) its momentum is controlled mainly by $2 k_{F}$ scattering processes across the Fermi surface edges along the Brillouin zone boundary (inset of Fig. 4). As shown in Figs. 4, 6, and 7 in Ref. 44, the first feature (a) cannot be captured by other promising types of $\mathrm{CO}$ obtained in the $t$ - $J$ model such as $s$-wave bond-charge order and flux phase whereas the $d$-wave bond-charge order can capture it. There are various $2 k_{F}$ scattering processes for the Fermi surface shown in the inset of Fig. 4. The reason why the scattering processed near $(\pi, 0)$ and $(0, \pi)$ is favored lies in the $d$-wave form factor, which is enhanced around $(\pi, 0)$ and $(0, \pi)$. In this sense, the feature $(\mathrm{b})$ is in line with the presence of a $d$-wave form factor. 


\section{SUMMARY}

Motivated by the recent RXS measurements of the doping dependence of the short-range CO observed in e-cuprates ${ }^{25}$, we studied the equal-time correlation function $S(\mathbf{q})$ associated with the $d$-wave bond-charge order by using a large- $N$ technique in the two-dimensional $t-J$ model. We extended our previous work $^{27}$ for a single doping rate by introducing the doping and temperature dependences of the damping $\Gamma$ and showed the consistency of our proposed scenario of the $d$-wave bond-charge order. The short-range CO is pronounced at low temperature (Figs. 3 and 5). The peak intensity develops gradually down to $\delta \approx 0.10$ and is substantially suppressed below that (Fig. 4), due to the strong damping effect presumably coming from antiferromagnetic fluctuations. A recent experiment on a h-cuprate ${ }^{55}$ also shows that the $\mathrm{CO}$ competes with antiferromagnetic fluctuations. The momentum of the short-range CO decreases with lowering doping and goes up below $\delta \approx 0.10$ (Fig. 4). All these features reasonably capture the essential features of the recent experimental data ${ }^{24}$. This agreement suggests three important implications for the physics in e-cuprates. (i) The origin of the CO lies in the magnetic exchange interaction, i.e., $J$-term in the $t$ - $J$ model. (ii) The CO is not generated by antiferromagnetic fluctuations. Rather they seem to contribute to the enhancement of the quasiparticle damping and consequently to suppress the charge ordering tendency. (iii) The CO is not a usual charge-density-wave, but a bond-charge order. In particular, it is characterized by $d$-wave symmetry and is connected to the nematic order in the limit of $\mathbf{q}=\mathbf{0}$. In this sense, the nematic physics plays a role also in e-cuprates, although so far it has been discussed only in h-cuprates.

\section{Acknowledgments}

The authors thank E. H. da Silva Neto, K. Ishii, B. Keimer, M. Minola, T. Tohyama, and R. Zeyher for very fruitful discussions. H.Y. acknowledges support by JSPS KAKENHI Grant Number JP15K05189. A.G. acknowledges the Japan Society for the Promotion of Science for a Short-term Invitational Fellowship program (S17027) under which this work was initiated. 


\section{Appendix A: $S(\mathrm{q})$ along the diagonal direction}

The $d$-wave bond-charge order in Fig. 2 occurs at $\mathbf{q}_{1}=\left( \pm q_{1}, \pm q_{1}\right) / \sqrt{2}$ and the instability at $\mathbf{q}_{2}=\left( \pm 2 \pi Q_{\mathrm{co}}, 0\right)$ and $\left(0, \pm 2 \pi Q_{\mathrm{co}}\right)$ is the second leading one. Nevertheless, the peak structure of $S(\mathbf{q})$ as well as $\chi_{d}(\mathbf{q})$ becomes sharper at $\mathbf{q}_{2}$ with decreasing $T$ whereas the spectrum around $\mathbf{q}_{1}$ is typically very broad and the peak structure develops only in the vicinity of the onset temperature of the charge instability. This peculiar feature was addressed in detail in Ref. 27. In the present paper, we introduced a large $\Gamma$. In this case, the peak structure around $\mathbf{q}_{1}$ is not realized unless the system is located in the vicinity of the phase boundary of the $d$-wave bond-charge order (dashed line in Fig. 2). To demonstrate this, we compute $S(\mathbf{q})$ for various choices of $\Gamma$ along the $(0,0)-(\pi, \pi)$ direction for $\delta=0.08$ at $T=0$ in Fig. 6 . There is a broad structure for a large $\Gamma$ and a small peak develops around $(0.75 \pi, 0.75 \pi)$ only near $\Gamma=0.025$, which is very close to the phase boundary. Since a large momentum near $\mathbf{q} \approx(\pi, \pi)$ is not accessible by RXS and furthermore the peak structure around $\mathbf{q}_{1}$ is not realized in general in the presence of a large $\Gamma$, we focus in this paper on the peak structure around $\mathbf{q}_{2}$, which is relevant to RXS as well as to recent experimental data ${ }^{24,25}$.

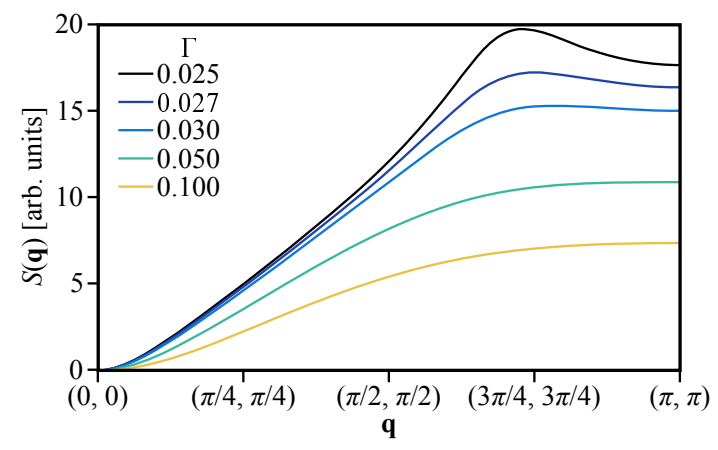

FIG. 6: $S(\mathbf{q})$ along the $(0,0)-(\pi, \pi)$ direction for various choices of $\Gamma$ at $T=0$ and $\delta=0.08$; the cutoff energy is $\omega_{c}=\infty$.

1 G. Ghiringhelli, M. Le Tacon, M. Minola, S. Blanco-Canosa, C. Mazzoli, N. B. Brookes, G. M. De Luca, A. Frano, D. G. Hawthorn, F. He, et al., Science 337, 821 (2012), URL http: //science. sciencemag. org/content/337/6096/821. 
2 J. Chang, E. Blackburn, A. T. Holmes, N. B. Christensen, J. Larsen, J. Mesot, R. Liang, D. A. Bonn, W. N. Hardy, A. Watenphul, et al., Nature Physics 8, 871 (2012), URL http: //dx.doi.org/10.1038/nphys2456.

3 A. J. Achkar, R. Sutarto, X. Mao, F. He, A. Frano, S. Blanco-Canosa, M. Le Tacon, G. Ghiringhelli, L. Braicovich, M. Minola, et al., Phys. Rev. Lett. 109, 167001 (2012), URL https://link.aps.org/doi/10.1103/PhysRevLett.109.167001.

4 E. Blackburn, J. Chang, M. Hücker, A. T. Holmes, N. B. Christensen, R. Liang, D. A. Bonn, W. N. Hardy, U. Rütt, O. Gutowski, et al., Phys. Rev. Lett. 110, 137004 (2013), URL https: //link.aps.org/doi/10.1103/PhysRevLett.110.137004.

5 S. Blanco-Canosa, A. Frano, E. Schierle, J. Porras, T. Loew, M. Minola, M. Bluschke, E. Weschke, B. Keimer, and M. Le Tacon, Phys. Rev. B 90, 054513 (2014), URL https: //link.aps.org/doi/10.1103/PhysRevB.90.054513.

6 R. Comin, A. Frano, M. M. Yee, Y. Yoshida, H. Eisaki, E. Schierle, E. Weschke, R. Sutarto, F. He, A. Soumyanarayanan, et al., Science 343, 390 (2014), URL http://science. sciencemag.org/content/343/6169/390.

7 E. H. da Silva Neto, P. Aynajian, A. Frano, R. Comin, E. Schierle, E. Weschke, A. Gyenis, J. Wen, J. Schneeloch, Z. Xu, et al., Science 343, 393 (2014), URL http://science. sciencemag.org/content/343/6169/393.

8 M. Hashimoto, G. Ghiringhelli, W.-S. Lee, G. Dellea, A. Amorese, C. Mazzoli, K. Kummer, N. B. Brookes, B. Moritz, Y. Yoshida, et al., Phys. Rev. B 89, 220511 (2014), URL https: //link.aps.org/doi/10.1103/PhysRevB.89.220511.

9 Y. Y. Peng, M. Salluzzo, X. Sun, A. Ponti, D. Betto, A. M. Ferretti, F. Fumagalli, K. Kummer, M. Le Tacon, X. J. Zhou, et al., Phys. Rev. B 94, 184511 (2016), URL https://link.aps . org/doi/10.1103/PhysRevB.94.184511.

10 S. Gerber, H. Jang, H. Nojiri, S. Matsuzawa, H. Yasumura, D. A. Bonn, R. Liang, W. N. Hardy, Z. Islam, A. Mehta, et al., Science 350, 949 (2015), URL http://science.sciencemag.org/ content/350/6263/949.

11 W. Tabis, B. Yu, I. Bialo, M. Bluschke, T. Kolodziej, A. Kozlowski, E. Blackburn, K. Sen, E. M. Forgan, M. v. Zimmermann, et al., Phys. Rev. B 96, 134510 (2017), URL https://link.aps . org/doi/10.1103/PhysRevB.96.134510.

12 B. Keimer, S. A. Kivelson, M. R. Norman, S. Uchida, and J. Zaanen, Nature 518, 179 (2015), 
URL http://dx.doi.org/10.1038/nature14165.

13 M. Bejas, A. Greco, and H. Yamase, Phys. Rev. B 86, 224509 (2012), URL https://link. aps.org/doi/10.1103/PhysRevB.86.224509.

14 S. Sachdev and R. La Placa, Phys. Rev. Lett. 111, 027202 (2013), URL https://link.aps . org/doi/10.1103/PhysRevLett.111.027202.

15 A. Allais, J. Bauer, and S. Sachdev, Phys. Rev. B 90, 155114 (2014), URL https://link . aps . org/doi/10.1103/PhysRevB.90.155114.

16 K. B. Efetov, H. Meier, and C. Pépin, Nature Physics 9, 442 (2013), URL http://dx.doi. org/10.1038/nphys2641.

17 H. Meier, C. Pépin, M. Einenkel, and K. B. Efetov, Phys. Rev. B 89, 195115 (2014), URL https://link.aps.org/doi/10.1103/PhysRevB.89.195115.

18 Y. Wang and A. Chubukov, Phys. Rev. B 90, 035149 (2014), URL https://link.aps.org/ doi/10.1103/PhysRevB.90.035149.

19 V. Mishra and M. R. Norman, Phys. Rev. B 92, 060507 (2015), URL https://link.aps.org/ doi/10.1103/PhysRevB.92.060507.

20 Y. Yamakawa and H. Kontani, Phys. Rev. Lett. 114, 257001 (2015), URL https://link.aps. org/doi/10.1103/PhysRevLett.114.257001.

21 W. A. Atkinson, A. P. Kampf, and S. Bulut, New Journal of Physics 17, 013025 (2015), URL http://stacks.iop.org/1367-2630/17/i=1/a=013025.

22 W. A. Atkinson, A. P. Kampf, and S. Bulut, Phys. Rev. B 93, 134517 (2016), URL https: //link.aps.org/doi/10.1103/PhysRevB.93.134517.

23 E. H. da Silva Neto, R. Comin, F. He, R. Sutarto, Y. Jiang, R. L. Greene, G. A. Sawatzky, and A. Damascelli, Science 347, 282 (2015), URL http://science.sciencemag.org/content/ $347 / 6219 / 282$.

24 E. H. da Silva Neto, B. Yu, M. Minola, R. Sutarto, E. Schierle, F. Boschini, M. Zonno, M. Bluschke, J. Higgins, Y. Li, et al., Science Advances 2, e1600782 (2016), URL http: //advances.sciencemag.org/content/2/8/e1600782.

25 E. H. da Silva Neto, M. Minola, B. Yu, W. Tabis, M. Bluschke, D. Unruh, H. Suzuki, Y. Li, G. Yu, D. Betto, et al., Phys. Rev. B 98, 161114 (2018), URL https://link.aps.org/doi/ 10.1103/PhysRevB.98.161114.

26 M. Bejas, A. Greco, and H. Yamase, New J. Phys. 16, 123002 (2014), URL http://stacks. 
iop.org/1367-2630/16/i=12/a=123002.

27 H. Yamase, M. Bejas, and A. Greco, Europhys. Lett. 111, 57005 (2015), URL https://doi. org/10.1209/0295-5075/111/57005.

28 Z.-X. Li, F. Wang, H. Yao, and D.-H. Lee, Phys. Rev. B 95, 214505 (2017), URL https: //link.aps.org/doi/10.1103/PhysRevB.95.214505.

29 Y. Mou and S. Feng, Philosophical Magazine 97, 3361 (2017), URL https://doi.org/10. $1080 / 14786435.2017 .1380319$.

30 The $d$-wave bond-charge order is referred to as various terms in literature, depending on its modulation vector $\mathbf{q}$ : kite state when $\mathbf{q}$ is close to $(\pi, \pi)$ in Refs. 56 and $57, d$-wave Pomeranchuk instability when $\mathbf{q}$ is close to $(0,0)$ in Refs. $13,33,34$, and 35 , and $\mathrm{BOP}_{x \bar{y}}$ for a general $\mathbf{q}$ in Ref. 26. The $d$-wave bond-charge order does not break time-reversal symmetry and thus should not be mixed with flux phase ${ }^{56,57}$, i.e., the so-called $d$-density wave ${ }^{58}$ or $d$-wave charge density wave $^{13}$, which breaks time-reversal symmetry. In the present paper we adopt the term of $d$-wave bond-charge order to emphasize the $d$-wave symmetry and bond character.

31 R. Comin, R. Sutarto, F. He, E. H. da Silva Neto, L. Chauviere, A. Fraño, R. Liang, W. N. Hardy, D. A. Bonn, Y. Yoshida, et al., Nature Materials 14, 796 (2015), article, URL http: //dx.doi.org/10.1038/nmat4295.

32 S. A. Kivelson, E. Fradkin, and V. J. Emery, Nature 393, 550 (1998), URL http://dx.doi. org/10.1038/31177.

33 H. Yamase and H. Kohno, J. Phys. Soc. Jpn. 69, 332 (2000), URL https://doi.org/10.1143/ JPSJ .69 .332$.

34 H. Yamase and H. Kohno, J. Phys. Soc. Jpn. 69, 2151 (2000), URL https://doi.org/10. 1143/JPSJ .69.2151.

35 C. J. Halboth and W. Metzner, Phys. Rev. Lett. 85, 5162 (2000), URL https://link.aps. org/doi/10.1103/PhysRevLett.85.5162.

36 V. Hinkov, D. Haug, B. Fauqué, P. Bourges, Y. Sidis, A. Ivanov, C. Bernhard, C. T. Lin, and B. Keimer, Science 319, 597 (2008), URL http://science.sciencemag.org/content/319/ $5863 / 597$.

37 R. Daou, J. Chang, D. LeBoeuf, O. Cyr-Choinière, F. Laliberté, N. Doiron-Leyraud, B. J. Ramshaw, R. Liang, D. A. Bonn, W. N. Hardy, et al., Nature 463, 519 (2010), URL http: //dx.doi.org/10.1038/nature08716. 
38 O. Cyr-Choinière, G. Grissonnanche, S. Badoux, J. Day, D. A. Bonn, W. N. Hardy, R. Liang, N. Doiron-Leyraud, and L. Taillefer, Phys. Rev. B 92, 224502 (2015), URL https://link. aps.org/doi/10.1103/PhysRevB.92.224502.

39 Y. Sato, S. Kasahara, H. Murayama, Y. Kasahara, E. G. Moon, T. Nishizaki, T. Loew, J. Porras, B. Keimer, T. Shibauchi, et al., Nature Physics 13, 1074 (2017), URL http://dx.doi.org/ $10.1038 /$ nphys 4205.

40 R. J. Gooding, K. J. E. Vos, and P. W. Leung, Phys. Rev. B 50, 12866 (1994), URL https: //link.aps.org/doi/10.1103/PhysRevB.50.12866.

41 G. B. Martins, J. C. Xavier, L. Arrachea, and E. Dagotto, Phys. Rev. B 64, 180513 (2001), URL https://link.aps.org/doi/10.1103/PhysRevB.64.180513.

42 A. Greco, H. Yamase, and M. Bejas, J. Phys. Soc. Jpn. 86, 034706 (2017), URL https://doi. org/10.7566/JPSJ .86.034706.

43 A. Greco, H. Yamase, and M. Bejas, Phys. Rev. B 94, 075139 (2016), URL https://link. aps.org/doi/10.1103/PhysRevB.94.075139.

44 M. Bejas, H. Yamase, and A. Greco, Phys. Rev. B 96, 214513 (2017), URL https://link. aps.org/doi/10.1103/PhysRevB.96.214513.

45 A. Greco, H. Yamase, and M. Bejas, Communications Physics 2, 3 (2019), ISSN 2399-3650, URL https://doi.org/10.1038/s42005-018-0099-z.

46 A. Foussats and A. Greco, Phys. Rev. B 70, 205123 (2004), URL https://link.aps.org/doi/ 10.1103/PhysRevB . 70.205123.

47 F. C. Zhang and T. M. Rice, Phys. Rev. B 37, 3759 (1988), URL https://link.aps.org/doi/ 10.1103/PhysRevB.37.3759.

48 H. Yamase, Phys. Rev. Lett. 93, 266404 (2004), URL https://link.aps.org/doi/10.1103/ PhysRevLett.93.266404.

49 A factor of $1 / 2$ comes from a large- $N$ formalism where $t$ is scaled by $t / N$ and the physical situation corresponds to $N=2$.

50 M. S. Hybertsen, E. B. Stechel, M. Schluter, and D. R. Jennison, Phys. Rev. B 41, 11068 (1990), URL https://link.aps.org/doi/10.1103/PhysRevB.41.11068.

51 In Fig. 3(d) in Ref. 27 this feature is less pronounced because the temperature dependence of the numerator in Eq. (3) was not taken into account.

52 K. Ishii, T. Tohyama, S. Asano, K. Sato, M. Fujita, S. Wakimoto, K. Tustsui, S. Sota, 
J. Miyawaki, H. Niwa, et al., Phys. Rev. B 96, 115148 (2017), URL https://link.aps.org/ doi/10.1103/PhysRevB.96.115148.

53 Stimulated by the marginal Fermi liquid theory ${ }^{59}$, one might wonder how our results are modified if $\Gamma$ depends also on $\omega$ by assuming $\Gamma \propto \max (T, \omega)$ at fixed density. In this case, the peak intensity and the peak position of $S(\mathbf{q})$ may change, but our conclusions are not modified.

54 E. M. Motoyama, G. Yu, I. M. Vishik, O. P. Vajk, P. K. Mang, and M. Greven, Nature 445, 186 (2007), URL http://dx.doi.org/10.1038/nature05437.

55 H. Jang, W.-S. Lee, S. Song, H. Nojiri, S. Matsuzawa, H. Yasumura, H. Huang, Y.-J. Liu, J. Porras, M. Minola, et al., Phys. Rev. B 97, 224513 (2018), URL https://link.aps.org/ doi/10.1103/PhysRevB.97.224513.

56 I. Affleck and J. B. Marston, Phys. Rev. B 37, 3774 (1988), URL https://link.aps.org/doi/ 10.1103/PhysRevB.37.3774.

57 E. Cappelluti and R. Zeyher, Phys. Rev. B 59, 6475 (1999), URL https://link.aps.org/ doi/10.1103/PhysRevB.59.6475.

58 S. Chakravarty, R. B. Laughlin, D. K. Morr, and C. Nayak, Phys. Rev. B 63, 094503 (2001), URL https://link.aps.org/doi/10.1103/PhysRevB.63.094503.

59 C. M. Varma, P. B. Littlewood, S. Schmitt-Rink, E. Abrahams, and A. E. Ruckenstein, Phys. Rev. Lett. 63, 1996 (1989), URL https://link.aps.org/doi/10.1103/PhysRevLett. 63.1996. 